\title{
Changes in corneal biomechanical properties after 24 hours of continuous intraocular pressure monitoring using a contact lens sensor
}

Laura Morales-Fernandez, MD, PhD, ${ }^{*}$ Javier Garcia-Bella, MD,*

Jose M. Martinez-de-la-Casa, MD, PhD, ${ }^{\dagger}$ Rubén Sanchez-Jean,

Federico Saenz-Frances, MD, PhD, ${ }^{*}$ Pedro Arriola-Villalobos, MD, PhD, ${ }^{*}$

Lucia Perucho, MD, ${ }^{*}$ Enrique Santos-Bueso, MD, PhD, ${ }^{*}$ Julian Garcia-Feijoo, MD, PhD ${ }^{\dagger, \ddagger}, \mathbb{S}$

ABSTRACT •

Objective: This study was designed to assess changes in corneal topography and biomechanics after intraocular pressure (IOP) monitoring using the Triggerfish contact lens sensor (CLS).

Methods: For this prospective study, 30 eyes of 30 subjects: 14 healthy subjects (G1) and 6 glaucoma patients (G2), were recruited for 24 hours of continuous IOP monitoring using the CLS. The following measurements were taken before CLS fitting and after lens removal: maximum keratometry (Kmax), mean keratometry (MK), and corneal astigmatism (Cyl) measured through Pentacam corneal topography, and the corneal biomechanical variables corneal hysteresis $(\mathrm{CH})$ and corneal resistance factor (CRF) measured with the Ocular Response Analyzer (ORA).

Results: Pentacam data revealed significant changes after CLS removal in Kmax $(+3.14 \pm 2.46 \mathrm{D}, p=0.002)$, MK (+0.52 $\pm 0.63 \mathrm{D}$, $p=0.02)$, and Cyl $(+0.48 \pm 0.53 \mathrm{D}, p=0.019)$ in $\mathrm{G} 1$; and $\mathrm{Kmax}(+1.38 \pm 1.43 \mathrm{D}, p=0.002)$ in $\mathrm{G} 2$. The changes observed were more pronounced in $\mathrm{G} 1$ than in $\mathrm{G} 2$ but differences were not significant. The ORA results indicated higher $\mathrm{CH}(11.35 \pm 2.42 \mathrm{vs}$ $8.17 \pm 2.09)$ and CRF $(10.3 \pm 2.03$ vs $9.1 \pm 1.81)$ before lens fitting in G1 than G2, while no significant changes were produced after CLS removal in either group.

Conclusions: The use of CLS for IOP monitoring over 24 hours caused topographic changes in both healthy subjects and glaucoma patients. No changes were produced in corneal biomechanics.

Glaucoma encompasses a group of eye diseases in which there is progressive damage to the optic nerve. Although the causes of glaucoma are thought to be multifactorial, intraocular pressure (IOP) is so far the only factor on which we can act efficiently, and its measurement is essential for diagnosing the disease as well as monitoring its progression and treatment.

Patients usually have their IOP measured in a single take during a routine visit to the doctor. However, rather than being a static variable, IOP fluctuates throughout the day following a circadian rhythm. ${ }^{1}$ Healthy subjects show a physiological increase in IOP at night of around $3 \mathrm{~mm}$ $\mathrm{Hg}$ to $6 \mathrm{~mm} \mathrm{Hg}^{2}$ In contrast, glaucoma patients show more pronounced fluctuation $(>10 \mathrm{~mm} \mathrm{Hg})$, and this is now considered an independent risk factor for disease progression. ${ }^{3}$ As a result, new devices have been developed to monitor IOP over a 24-hour period to assess the risk of glaucoma progression. ${ }^{4}$

The SENSIMED Triggerfish contact lens sensor (CLS [Sensimed AG, Lausanne, Switzerland]) is a silicone contact lens with an embedded sensor that allows for outpatient IOP monitoring while patients carry on with their daily life activities. The CLS contains 2 titanium-platinum strain gauges (wire loops) that detect fluctuations in the diameter of the corneoscleral junction and establish any correlation between changes in volume and IOP. ${ }^{5}$ The device takes measurements over 30 seconds every 5 minutes to generate a total of 288 measurements in a 24-hour period. The lens transmits information through its antenna, which is battery powered. Once the lens is removed, the data are transferred from the recorder via a Bluetooth adapter to a computer for analysis. Measures from the sensor are in electrical units (millivolts) and are visualized graphically as IOP curves using an arbitrary unit of measure (millivolt equivalents, $\mathrm{mV}$ eq).

The device has demonstrated fair reproducibility of measurements over repeated continuous 24-hour IOP monitoring. ${ }^{6}$ The reliability and precision of the record obtained depends largely on correct adaptation of the CLS to the ocular surface. Because of this, different lens sizes are available, and these are fitted according to the subject's radius of corneal curvature (curvature radii of 8.4, 8.7, and $9 \mathrm{~mm}$ for steep, medium, and flat, respectively). Other factors may also affect measurement precision. In several reports, changes in corneal thickness ${ }^{7}$ and corneal curvature have been described after IOP monitoring with this

\footnotetext{
(C) 2017 Published by Elsevier Inc on behalf of the

Canadian Ophthalmological Society.

https://doi.org/10.1016/j.jcjo.2017.10.028

ISSN 0008-4182/17
}

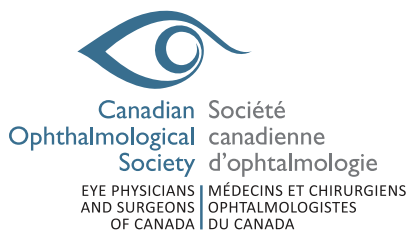




\section{Changes in corneal biomechanics after IOP monitoring-Morales-Fernandez et al.}

sensor. However, most reports have provided data for young healthy subjects. ${ }^{8}$ On the other hand, $\mathrm{CH}$ and CRF have been found to be reduced in contact lens wearers. These corneal biomechanical variables are related to the presence and progression of glaucoma. ${ }^{10}$ But no studies have been previously conducted analyzing the impact of CLS wear on the corneal biomechanics in glaucoma patients.

The present study was designed to assess whether IOP monitoring with this device over a 24-hour period would produce changes in corneal curvature and hysteresis both in healthy subjects and glaucoma patients.

\section{MATERIALS AND METHOdS}

Thirty SENSIMED Triggerfish contact lens sensors were available. We therefore recruited 30 subjects for this prospective study, 16 of whom were glaucoma patients. The glaucoma patients were managed at the Glaucoma Department of the Hospital Clinico San Carlos (Madrid, Spain), and inclusion criteria were a diagnosis of primary open angle glaucoma (POAG) and no previous glaucoma surgery. The remaining participants assigned to the healthy subject group were also recruited among the staff and outpatients of our center. Before enrollment, these individuals underwent a comprehensive ophthalmologic examination, including visual acuity, refractive status, and a dilated fundus examination by the same examiner (LMF) to exclude glaucoma suspects.

General inclusion criteria were a flat radius of curvature of $7.25 \mathrm{~mm}$ to $8.25 \mathrm{~mm}$, so that all participants could be fitted with the medium-sized CLS, following the manufacturer's guidelines.

In order to minimize the effect of refractive error on the study, eyes with a spherical equivalent greater than \pm 6 diopters were excluded.

In each participant, one eye was randomly selected for inclusion in the study, using a randomization list obtained from www.randomization.com.

Written informed consent was obtained from each participant. The study protocol received Institutional Review Board approval and adhered to the tenets of the 1964 Declaration of Helsinki.

In the selected eye of each participant, a medium-sized CLS lens was fitted at 2:00 PM and removed at the same time on the following day. All study participants underwent an eye examination before and after wearing the lens, including objective refraction using the Humphrey 599 autorefractometer (Humphrey Instruments Inc, Carl Zeiss Group, Jena, Germany), biomicroscopy, Pentacam corneal topography (Oculus Inc, Berlin, Germany), and IOP measurement. The IOP was obtained first with the Ocular Response Analyzer (ORA, Reichert Technologies, Depew, N.Y.) and then using Perkins Handheld Applanation Tonometer (Perkins; Clement-Clarke, Columbus, Ohio).
Three measurements were taken with each device, and the means entered in the statistical analysis. The Pentacam measurements made were central corneal thickness (CCT), thinnest central cornea (TCC), anterior chamber volume (ACV), maximum keratometry (Kmax), mean keratometry $(\mathrm{MK})$, and corneal astigmatism $(\mathrm{Cyl})$. ORA was used to determine corneal-compensated IOP (IOPcc), Goldmann-correlated IOP (IOPg), corneal hysteresis $(\mathrm{CH})$, and corneal resistance factor (CRF).

The Student's $t$ test was used to compare means. $\chi^{2}$ tests were used for the analysis of dichotomous variables. All statistical tests were performed using the SPSS package version 18.0 (SPSS Inc, Chicago, Ill.). Significance was set at $p<0.05$.

\section{Results}

The baseline demographics of the 2 study groups are provided in Table 1 . The 14 healthy subjects in group 1 (G1) were of mean age $22.40 \pm 2.31$ years (range 21 to 43 years). The 16 glaucoma patients in group 2 (G2) were of mean age $74.81 \pm 6.72$ years (range 52 to 88 years). In the patient group (G2), mean number of eye drops per eye was $1.1 \pm 0.6$ per day. Ten patients were treated with topical prostaglandins (latanoprost eye drops), 3 patients with topical beta-blockers (timolol $0.5 \%$ ), and 3 patients with a combination therapy (timolol/brinzolamide).

No significant difference was produced in Perkins tonometry IOP measurements before lens fitting and after lens removal in both G1 $(13.27 \pm 2.57 \mathrm{~mm} \mathrm{Hg}$ vs 12.18 $\pm 2.75 \mathrm{~mm} \mathrm{Hg}, p=0.082)$ and G2 $(19.67 \pm 2.73 \mathrm{~mm}$ $\mathrm{Hg}$ vs $19.70 \pm 5.41 \mathrm{~mm} \mathrm{Hg}, p=0.976)$.

The Pentacam measurements made in both groups before and after lens wear are provided in Table 2. After lens removal, Kmax increases of $+3.14 \pm 2.46 \mathrm{D}$ and $+1.38 \pm 1.43 \mathrm{D}$ were observed in G1 and G2, respectively $(p<0.05$; an example is shown in Fig. 1).

The ORA data recorded (Table 3) failed to vary before and after lens wear in either group. Notwithstanding, changes in G2 were more pronounced, and reductions of $-0.55 \pm 1.5(8.17 \pm 2.09$ vs $7.61 \pm 1.97, p=0.176)$ and $-0.34 \pm 1.03(9.1 \pm 1.81$ vs $8.88 \pm 2.10, p=0.352)$ in $\mathrm{CH}$ and $\mathrm{CFR}$, respectively, were observed in response to the lens, although without significance.

\begin{tabular}{|c|c|c|c|}
\hline \multicolumn{4}{|c|}{$\begin{array}{l}\text { Table 1-Demographics and ocular characteristics of the } \\
\text { study participants }\end{array}$} \\
\hline & $\begin{array}{l}\text { G1 }(n=14) \\
\text { Mean (SD) }\end{array}$ & $\begin{array}{l}\text { G2 }(n=16) \\
\text { Mean (SD) }\end{array}$ & $p$ \\
\hline Sex (male:female) & $6: 8$ & $7: 9$ & 0.112 \\
\hline Age (years) & $22.40(2.3)$ & $74.81(6.72)$ & $0.013^{\dagger}$ \\
\hline Flat radius curvature $(\mathrm{mm})$ & $7.95(0.16)$ & $7.82(0.34)$ & $0.173^{\dagger}$ \\
\hline \multicolumn{4}{|c|}{$\begin{array}{l}\text { G1, healthy subjects; G2, glaucoma group. } \\
{ }^{*} \chi^{2} \text { test } \\
{ }^{\dagger} \text { Student's } t \text { test. }\end{array}$} \\
\hline
\end{tabular}


Changes in corneal biomechanics after IOP monitoring-Morales-Fernandez et al.

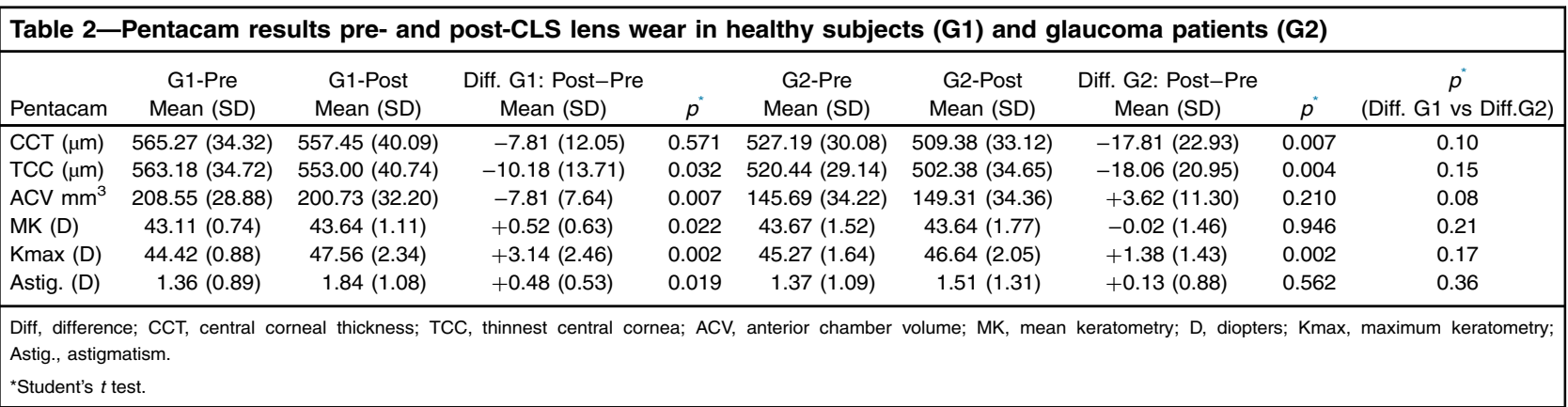

Post-hoc power was calculated (39.93\%) to detect a $\mathrm{CH}$ difference between both groups equal or greater than 0.8 , considering that our sample size was 16 patients in the glaucoma group and 14 subjects in the control group (assuming an alpha risk of 95\%). ${ }^{11}$

\section{Discussion}

The SENSIMED Triggerfish contact lens device has been described as a useful tool for continuous monitoring of IOP over a 24-hour period in outpatients. The tolerance and safety of this device has been described, highlighting its main effects of superficial corneal staining and conjunctival hyperemia. ${ }^{6}$ However, recent studies have detected an increase in CCT and corneal curvature after monitoring of IOP using this device.

The impacts of silicone contact lenses on corneal thickness have been well described in the literature. ${ }^{12,13}$ Corneal swelling occurs when less oxygen reaches the cornea through the closed eyelids during sleep, causing a physiological increase in corneal thickness. ${ }^{14}$ Hydrogel silicone contact lenses show high oxygen permeability, minimizing these overnight effects on corneal thickness $(0.7 \%-1.4 \%) .{ }^{15}$ CLS use has been associated with greater corneal swelling $(4.4 \pm 1.7 \%)$, probably because CLS is made of pure silicone. ${ }^{8}$ This increase in CCT has been observed only at night and resolves rapidly with blinking. In our study participants who wore the device over a 24hour period from 2:00 PM until the same time the following day, this increase was not noted.

One of the objectives of our study was to examine possible corneal curvature changes using the Pentacam. We hypothesized that the lens would cause peripheral corneal applanation and central curving, detectable as elevated topographic dimensions. Hubanova et al. ${ }^{8}$ revealed a significant increase in corneal irregularities in healthy, young patients in response to CLS wear mainly within the central $3 \mathrm{~mm}$ and $5 \mathrm{~mm}$ of the cornea. However, although they observed an increase in corneal curvature, MK values as measured with Orbscan did not vary significantly.

In our study, significant differences between measurements taken before and after 24 hours of lens wear were observed across practically all topographical variables in G1. On the contrary, however, patients in G2 showed significant differences only in Kmax. Changes in MK and Cyl tended to be more pronounced in the healthy subjects than glaucoma patients, although the comparison between the differences in both groups lacked significance. The majority of studies on the side effects of 24-hour IOP monitoring have examined healthy subjects aged 20 to 30 years. ${ }^{5,16}$ The average age of the control group in our study is similar to that of these other studies. However, our patient group was much older since POAG typically affects older patients. ${ }^{17}$ Hence, the greater increase in corneal curvature observed here in the group of healthy subjects is likely attributable to the increase in corneal stiffness that occurs with age, which makes it less susceptible to deformity.

Another objective of our study was to determine the potential effects of CLS wear on the corneal biomechanical variables $\mathrm{CH}$ and $\mathrm{CRF}$.

$\mathrm{CH}$ is a measure of the cornea's viscous damping capacity associated with its stiffness or elasticity, and CRF is an indicator of the overall resistance of the cornea. These biomechanical properties of the cornea change as we age. However, $\mathrm{CH}$ is lower in patients with glaucoma and normal-pressure glaucoma than in healthy subjects, perhaps indicating a lower tissue damping capacity in glaucoma. In effect, a low $\mathrm{CH}$ may be considered an independent indicator of the presence and progression of glaucoma. ${ }^{10,18}$ Recently, however, Radaie-Moghadam et al. ${ }^{9}$ reported a decreased $\mathrm{CH}$ and CRF in contact lens wearers. Thus, lens wear may cause artifacts in readings or interfere with the correct monitoring of IOP and this could also occur with a CLS lens, although we did not observe this in our study, probably because of the short period of lens wear.

Before lens fitting, variables associated with IOP (IOPcc and IOPg) were higher in the glaucoma group, as expected, while variables associated with corneal biomechanics $(\mathrm{CH}$ and $\mathrm{CRF}$ ) were higher in the healthy individuals. This reflects the lower $\mathrm{CH}$ and $\mathrm{CRF}$ in glaucoma patients when compared with the control group $(8.17 \pm 2.09$ vs 11.35 and $9.1 \pm 1.81$ vs $10.3 \pm 2.03$, respectively). But significant changes in corneal biomechanical properties in response to 24 hours of IOP monitoring were not observed. Mansouri et al. $^{6}$ reported fair to good reproducibility of the recorded IOP patterns in glaucoma patients, suggesting that output signal of the 
Changes in corneal biomechanics after IOP monitoring-Morales-Fernandez et al.

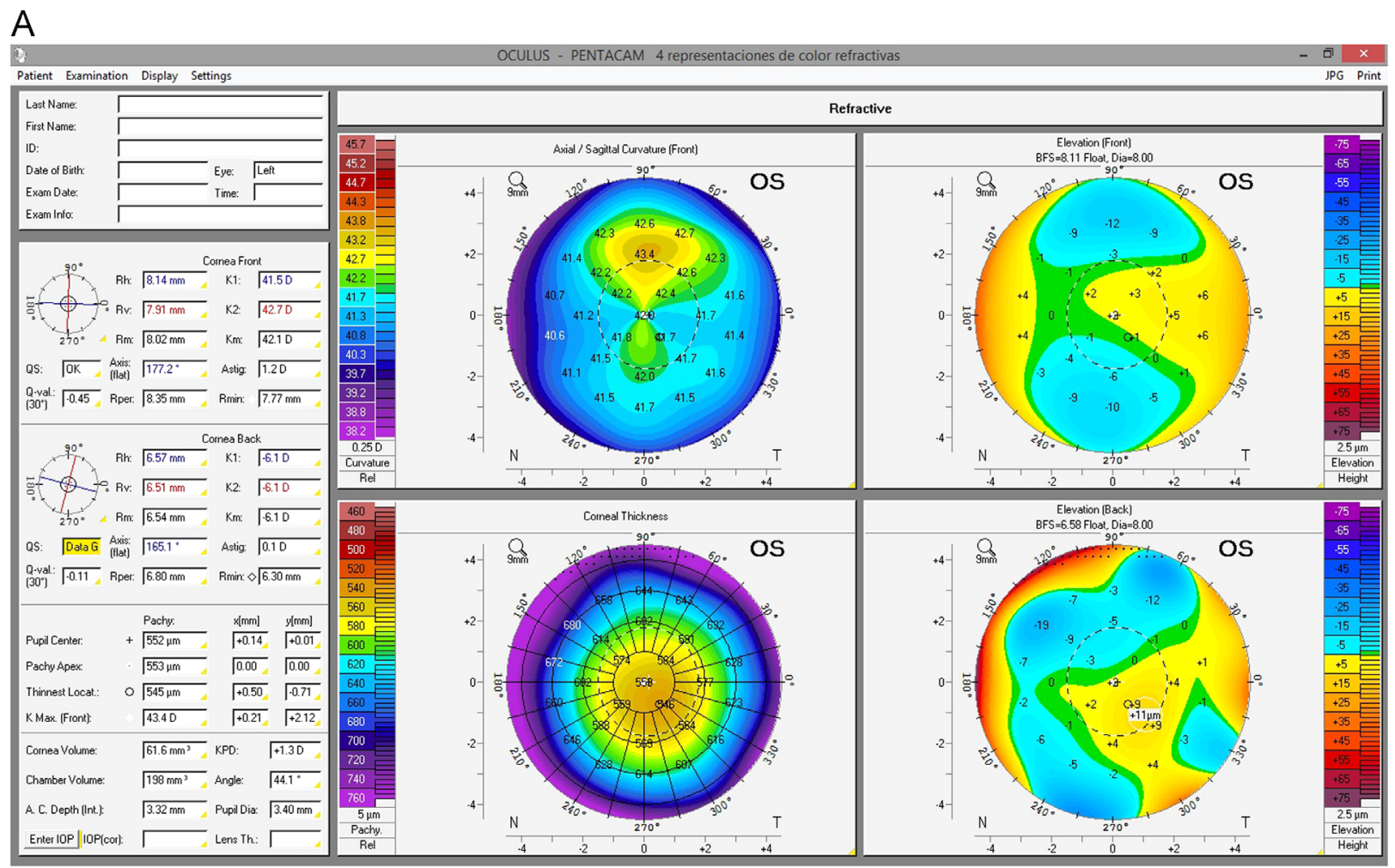

B

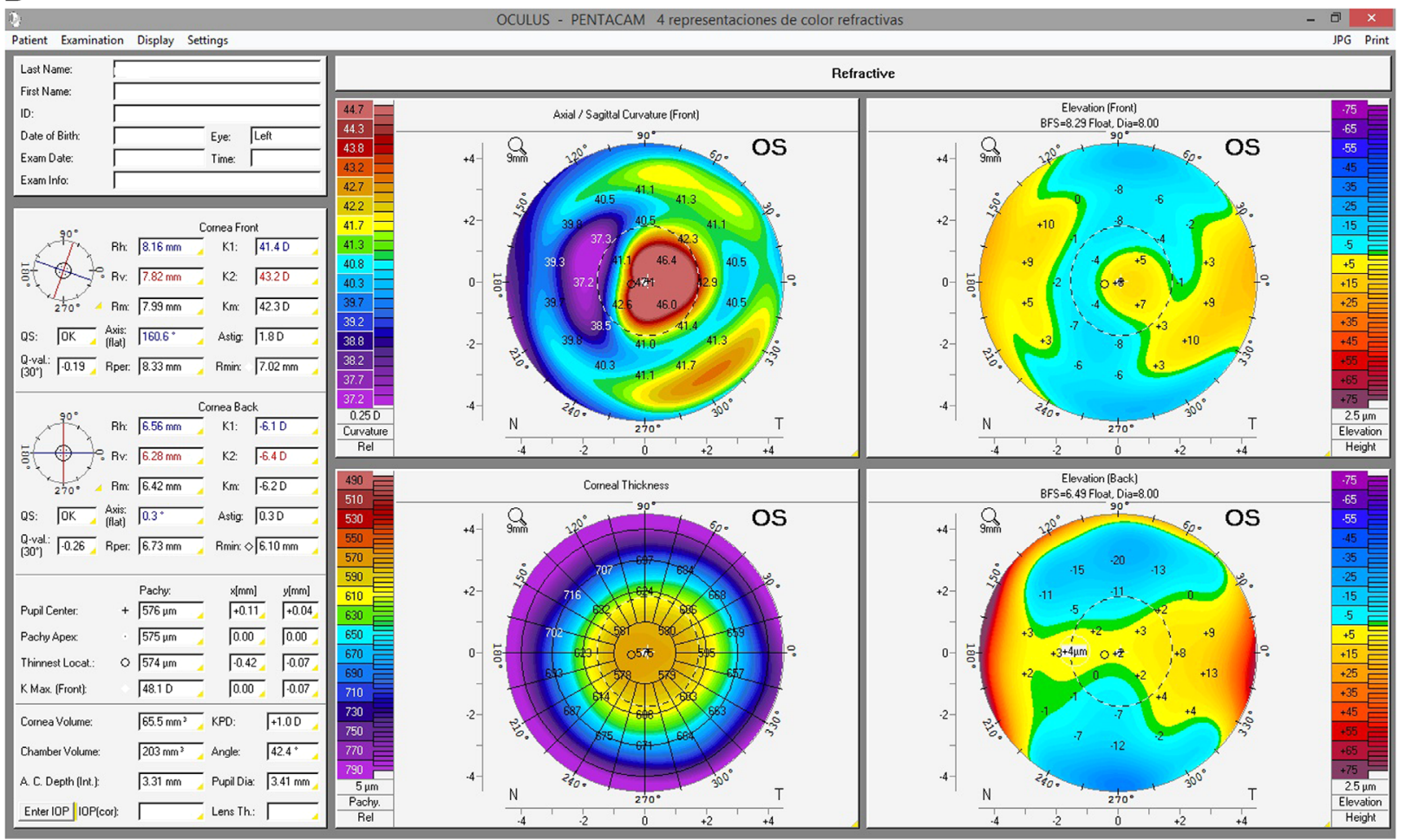

Fig. 1-Visual representation of the changes observed in corneal topography using Pentacam after continuous 24-hour IOP monitoring. A, Before CLS lens wear. B, After CLS wear.

CLS can be subject to artifacts such as changes in corneal shape and thickness. However, these potential artifacts are not yet well defined, and changes in biomechanical corneal properties could be one of them. Our results revealed no statistical differences in $\mathrm{CH}$ and CRF after 24-hour IOP monitoring with CLS. Despite this, our study found low statistical power and we could not analyze the correlation between biomechanical corneal properties and the 
Changes in corneal biomechanics after IOP monitoring-Morales-Fernandez et al.

\begin{tabular}{|c|c|c|c|c|c|c|c|c|c|}
\hline ORA & $\begin{array}{c}\text { G1-Pre } \\
\text { Mean (SD) }\end{array}$ & $\begin{array}{c}\text { G1-Post } \\
\text { Mean (SD) }\end{array}$ & $\begin{array}{l}\text { Diff. G1: Post-Pre } \\
\text { Mean (SD) }\end{array}$ & $\mathrm{P}^{*}$ & $\begin{array}{c}\text { G2-Pre } \\
\text { Mean (SD) }\end{array}$ & $\begin{array}{c}\text { G2-Post } \\
\text { Mean (SD) }\end{array}$ & $\begin{array}{l}\text { Diff. G2: Post-Pre } \\
\text { Mean (SD) }\end{array}$ & $p^{*}$ & $\begin{array}{c}p^{*} \\
\text { (Diff.G1 vs Diff.G2) }\end{array}$ \\
\hline IOPcc $(\mathrm{mm} \mathrm{Hg})$ & $12.14(4.27)$ & 11.34 (3.19) & $-0.8(2.66)$ & 0.337 & $20.05(4.46)$ & $21.43(6.49)$ & $+1.38(4.33)$ & 0.237 & 0.31 \\
\hline $\mathrm{IOPg}(\mathrm{mm} \mathrm{Hg})$ & $12.26(3.42)$ & $11.27(3.22)$ & $-0.99(2.75)$ & 0.259 & 17.39 (3.87) & $18.30(6.37)$ & $+0.91(4.33)$ & 0.431 & 0.18 \\
\hline $\mathrm{CH}$ & $11.35(2.42)$ & $11.36(2.65)$ & $+0.18(0.85)$ & 0.945 & 8.17 (2.09) & 7.61 (1.97) & $-0.55(1.5)$ & 0.176 & 0.13 \\
\hline CRF & $10.30(2.03)$ & $9.95(2.75)$ & $-0.25(0.95)$ & 0.785 & $9.10(1.81)$ & $8.88(2.10)$ & $-0.34(1.03)$ & 0.352 & 0.67 \\
\hline
\end{tabular}

reliability and precision of the record, so new studies are required to dismiss the influence of $\mathrm{CH}$ and $\mathrm{CRF}$ on the data recorded by the sensor.

The main limitation of this preliminary study was the large age difference between groups. However, we were still able to compare our results with those reported by others. Another limitation was the small number of participants conferring a low statistical power to any differences observed. In future studies, the impacts of 24-hour IOP monitoring should be addressed in a larger study population, and it would also be interesting to assess the effects of corneal curvature changes on the reliability of the data provided by the CLS sensor.

In conclusion, our findings indicate that the use of a CLS lens to continuously monitor IOP over a 24-hour period causes traceable changes in corneal curvature, producing a significant increase in the central region that affects healthy subjects more than glaucoma patients. No impacts of CLS use on corneal biomechanical properties (CH and $\mathrm{CFR}$ ) were observed.

\section{References}

1. Barkana Y, Anis S, Liebmann J, Tello C, Ritch R. Clinical utility of intraocular pressure monitoring outside of normal office hours in patients with glaucoma. Arch Ophthalmol. 2006;124:793-7.

2. Grippo TM, Liu JH, Zebardast N, Arnold TB, Moore GH, Weinreb RN. Twenty-four-hour pattern of intraocular pressure in untreated patients with ocular hypertension. Invest Ophthalmol Vis Sci. 2013;54:512-7.

3. Deokule SP, Doshi A, Vizzeri G, et al. Relationship of the 24-hour pattern of intraocular pressure with optic disc appearance in primary open-angle glaucoma. Ophthalmology. 2009;116:833-9.

4. Leonardi M, Leuenberger P, Bertrand D, Bertsch A, Renaud P. First steps toward noninvasive intraocular pressure monitoring with a sensing contact lens. Invest Ophthalmol Vis Sci. 2004;45:3113-7.

5. De Smedt S, Mermoud A, Schnyder C. 24-hour intraocular pressure fluctuation monitoring using an ocular telemetry Sensor: tolerability and functionality in healthy subjects. J Glaucoma. 2012;21:539-44.

6. Mansouri K, Medeiros FA, Tafreshi A, Weinreb RN. Continuous 24-hour monitoring of intraocular pressure patterns with a contact lens sensor: safety, tolerability, and reproducibility in patients with glaucoma. Arch Ophthalmol. 2012;130:1534-9.

7. Freiberg FJ, Lindell J, Thederan LA, Leippi S, Shen Y, Klink T. Corneal thickness after overnight wear of an intraocular pressure fluctuation contact lens sensor. Acta Ophthalmol. 2012;90:e534-9.

8. Hubanova R, Aptel F, Chiquet C, Mottet B, Romanet JP. Effect of overnight wear of the Triggerfish ${ }^{\circledR}$ sensor on corneal thickness measured by Visante ${ }^{\circledR}$ anterior segment optical coherence tomography. Acta Ophthalmol. 2014;92:e119-23.
9. Radaie-Moghadam S, Hashemi H, Jafarzadehpur E, Yekta AA, Khabazkhoob M. Corneal biomechanical changes following toric soft contact lens wear. J Ophthalmic Vis Res. 2016;11:131-5.

10. Terai N, Raiskup F, Haustein M, Pillunat LE, Spoerl E. Identification of biomechanical properties of the cornea: the ocular response analyzer. Curr Eye Res. 2012;37:553-62.

11. Ayala M. Corneal hysteresis in normal subjects and in patients with primary open-angle glaucoma and pseudoexfoliation glaucoma. Ophthalmic Res. 2011;46:187-91.

12. du Toit TR, Vega JA, Fonn D, Simpson T. Diurnal variation of corneal sensitivity and thickness. Cornea. 2003;22:205-9.

13. Wang J, Fonn D, Simpson TL. Topographical thickness of the epithelium and total cornea after hydrogel and PMMA contact lens wear with eye closure. Invest Ophthalmol Vis Sci. 2003;44:1070-107.

14. Martin R, de Juan V, Rodriguez G, Cuadrado R, Fernandez I. Measurement of corneal swelling variations without removal of the contact lens during extended wear. Invest Ophthalmol Vis Sci. 2007; $48: 3043-50$.

15. Steffen RB, Schnider CM. The impact of silicone hydrogel materials on overnight corneal swelling. Eye Contact Lens. 2007;33:115-20.

16. Mottet B, Aptel F, Romanet JP, Hubanova R, Pepin JL, Chiquet C. 24-hour intraocular pressure rhythm in young healthy subjects evaluated with continuous monitoring using a contact lens sensor. JAMA Ophthalmol. 2013;131:1507-16.

17. Quigley HA, Broman AT. The number of people with glaucoma worldwide in 2010 and 2020. Br J Ophthalmol. 2006;90:262-7.

18. Johannesson G, Hallberg P, Ambarki K, Eklund A, Lindén C. Agedependency of ocular parameters: a cross sectional study of young and elderly healthy subjects. Graefes Arch Clin Exp Ophthalmol. 2015;253:1979-83.

\section{Footnotes and Disclosure:}

This study was conducted with the cooperation of the company Sensimed AG (Lausanne, Switzerland).

From the * Ophthalmology Unit, Hospital Clinico San Carlos, Faculty of Medicine, Universidad Complutense de Madrid, Instituto de Investigacion Sanitaria del Hospital Clinico San Carlos (IdISSC), Madrid, Spain; †Department of Ophthalmology and ORL, Faculty of Medicine, Universidad Complutense de Madrid, Instituto de Investigacion Sanitaria del Hospital Clinico San Carlos (IdISSC), Madrid, Spain; ‡Instituto de Investigaciones Oftalmologicas Ramon Castroviejo, Universidad Complutense de Madrid, Madrid, Spain; \$Cooperative Research Network on Age-Related Ocular Disease, and Visual and Life Quality, Instituto de Salud Carlos III, Madrid, Spain.

Originally received Apr. 17, 2017. Final revision Oct. 5, 2017. Accepted Oct. 10, 2017.

Correspondence to Laura Morales-Fernandez, Profesor Martin Lago SN, Madrid 28009; lauramoralesfernandez@gmail.com 